\title{
Current status of submucosal tunneling endoscopic resection for gastrointestinal submucosal tumors originating from the muscularis propria layer (Review)
}

\author{
YUYONG TAN, JIRONG HUO and DELIANG LIU \\ Department of Gastroenterology, The Second Xiangya Hospital of Central \\ South University, Changsha, Hunan 410011, P.R. China
}

Received June 22, 2016; Accepted July 7, 2017

DOI: $10.3892 / 01.2017 .6869$

\begin{abstract}
Gastrointestinal submucosal tumors (SMTs) have been increasingly identified via the use of endoscopic ultrasonography, and removal is often recommended for SMTs that are $>2 \mathrm{~cm}$ in diameter or symptomatic. Submucosal tunneling endoscopic resection (STER), also known as submucosal endoscopic tumor resection, endoscopic submucosal tunnel dissection or tunneling endoscopic muscularis dissection, is a novel endoscopic technique for treating gastrointestinal SMTs originating from the muscularis propria layer, and has been demonstrated to be effective in the removal of SMTs with a decreased rate of recurrence by clinical studies. STER may be performed for patients with esophageal or cardia SMTs, and its application has expanded beyond these types of SMTs due to modifications to the technique. The present study reviewed the applications, procedure, efficacy and complications associated with STER.
\end{abstract}

\section{Contents}

1. Introduction

2. Preoperative assessment

3. Applications of STER

4. STER procedure

Correspondence to: Dr Deliang Liu, Department of Gastroenterology, The Second Xiangya Hospital of Central South University, 139 Middle Renmin Road, Changsha, Hunan 410011, P.R. China

E-mail: liudeliang@medmail.com.cn

Abbreviations: SMT, submucosal tumor; EUS, endoscopic ultrasonography; ESD, endoscopic submucosal dissection; ESE, endoscopic submucosal excavation; STER, submucosal tunneling endoscopic resection; MP, muscularis propria

Key words: submucosal tumor, gastrointestinal gastrointestinal surgical procedures, submucosal tunneling endoscopic resection
5. Efficacy of STER

6. Complications of STER

7. Conclusions

\section{Introduction}

Following the identification of a gastrointestinal submucosal tumor (SMT), periodic surveillance using endoscopy and endoscopic ultrasonography (EUS) remains a major strategy, but the use of this strategy is associated with multiple concerns, including patient compliance and stress, cost-effectiveness, and the risks associated with repeated endoscopic procedures and delayed diagnosis of malignancy $(1,2)$. Furthermore, certain tumors exhibit malignant potential, particularly those that originate from the muscularis propria (MP) layer or are large in diameter (1). Therefore, removing these SMTs is crucial. Current methods to remove SMTs include surgery and endoscopic resection, compared with the latter, surgical approaches are more invasive and associated with increased costs and a longer hospital stay. Endoscopic resection is a first-line treatment for SMTs $\leq 50 \mathrm{~mm}$ in diameter $(1,2)$. Alternative methods include endoscopic submucosal dissection (ESD), endoscopic submucosal excavation (ESE) and endoscopic full-thickness resection, but these may be associated with unsatisfactory outcomes due to incomplete resection and/or the risk of perforation during the procedure (3-5). Submucosal tunneling endoscopic resection (STER) has emerged as a novel technique for treating upper gastrointestinal SMTs and has yielded promising results (6-17). STER possesses multiple advantages over other endoscopic methods, including the maintenance of mucosal integrity, the facilitation of an increased rate of healing and a decreased risk of pleural/abdominal infection. In addition, the submucosal tunnel helps to maintain a clear visual field, which facilitates an improved response to intraoperative bleeding. The present study summarized the current status of STER, including its applications, procedure, efficacy and complications.

\section{Preoperative assessment}

Prior to performing STER, the presence, originating layer, size, and the presence or absence of malignancy-associated 
risk features of the SMT should be confirmed. The SMT should also be distinguished from extrinsic compression or hemangioma. Esophagogastroduodenoscopy and colonoscopy may be used to locate the lesion, and EUS and computerized tomography (CT) may be used to determine the originating layer, size and risk features of the SMT (1).

\section{Applications of STER}

STER for esophageal and cardia SMTs $\leq 35 \mathrm{~mm}$. STER is a complicated procedure with a decreased space for operation in the submucosal tunnel, and therefore was initially performed for esophageal and cardia SMTs, with most researchers recommending a maximum resectable lesion size of $35 \mathrm{~mm}$ (6-10). With STER being increasingly applied for patients with multiple types of SMT, STER has been modified multiple times and its application has expanded further. The patient selection diagram for candidates of STER at the Second Xiangya Hospital of Central South University (Changsha, China) was provided (Fig. 1).

STER for gastric SMTs. The stomach possesses specific anatomical and physiological features, including a large lumen, increased flexibility, an unfixed position and thick mucosa, that render generating a submucosal tunnel more challenging compared with doing so in the esophagus, and not all gastric SMTs are suitable for STER. In addition to those in the cardia, STER may be used as a treatment for SMTs located in the gastric corpus or fundus proximate to the cardia, the lesser curvature of the gastric corpus and the greater curvature of the gastric antrum. Lu et al (18) treated 18 patients with gastric fundus SMTs using STER; 19 tumors were removed, en bloc resection was achieved for all the patients and the mean tumor size was $21 \mathrm{~mm}$ (range, 8-50 mm). Lu et al (19) treated 45 patients with gastric SMTs using STER, 43 cases were successfully treated and 47 tumors were removed. The SMTs were all located in the cardia, the gastric fundus proximate to the cardia or the gastric antrum. En bloc resection was achieved for all the patients and the mean tumor size was $14 \mathrm{~mm}$ (range, 5-50 mm). Li et al (20) reported on 32 patients with gastric SMTs who were treated using STER without severe complications. Of these SMTs, 12 were located in the gastric corpus proximate to the cardia, 3 in the gastric fundus proximate to the cardia, 6 in the lesser curvature of the gastric corpus and 11 in the greater curvature of the gastric antrum. En bloc resection was achieved for all the patients and the mean tumor size was $23 \mathrm{~mm}$ (range, $10-50 \mathrm{~mm}$ ).

STER for multiple SMTs. Although the majority of the SMTs in the MP layer are solitary, multiple studies have reported the presence of multiple SMTs $(11,13,18,19,21,22)$. Chen et al (21) reported a patient simultaneously exhibiting esophageal and cardia SMT, and the two SMTs were successfully removed using STER with a single tunnel. Zhang et al (22) treated 23 patients with multiple SMTs in the upper gastrointestinal tract using STER. A total of 49 SMTs were removed and 3 of the patients exhibited three coexisting tumors.

STER for esophageal and cardia SMTs $>35 \mathrm{~mm}$. Although the majority of researchers recommended a maximum resectable lesion size of $35 \mathrm{~mm}$ during STER due to the decreased space for operation in the submucosal tunnel, STER has been applied multiple times for SMTs $>35 \mathrm{~mm}$, with the largest SMT reported to undergo STER, to the best of our knowledge, being $70 \mathrm{~mm}$ (23-29). Wang et al (15) retrospectively analyzed the clinical data of 80 patients with a total of 83 SMTs, 70 of which were $\leq 35 \mathrm{~mm}$ and 13 of which were $>35 \mathrm{~mm}$, and demonstrated that STER resulted in a similar efficacy and rate of complications for SMTs $\leq 35 \mathrm{~mm}$ and those $>35 \mathrm{~mm}$, although an increased operative duration was demonstrated for the latter compared with the former.

STER for rectal SMTs. The rectum possesses a thin mucosa and a tortuous lumen, thereby rendering the generation of a submucosal tunnel more challenging compared with doing so in the esophagus. To the best of our knowledge, only one center has reported the use of STER in patients with rectal SMTs. Hu et al (30) treated 12 patients with rectal SMTs using STER; en bloc resection was achieved for all the patients and the median size of the resected tumors was $14 \mathrm{~mm}$ (range, 10-30 mm). No severe complications were detected and no recurrence was revealed during the 4-33 month follow up.

\section{STER procedure}

The STER procedure in The Second Xiangya Hospital of Central South University (Fig. 2) is typically performed with the patient in the supine or lateral position under general anesthetic and with the airway intubated. $\mathrm{CO}_{2}$ insufflation is recommended (29).

Identification of the tumor. The tumor is identified and accurately located. For SMTs that are challenging to locate, including SMTs proximate to the fundus of the stomach, the submucosal injection of indigo carmine or methylene blue may be performed to help locate the tumor and guide the direction of subsequent tunneling (20).

Submucosal injection. A fluid cushion is subsequently generated through a submucosal injection consisting of saline solution with indigo carmine $3-5 \mathrm{~cm}$ from the SMT. Typically, epinephrine is added to the solution to decrease the risk of intraoperative bleeding. For rectal or gastric SMTs, the submucosal injection is performed 2-3 cm from the SMT $(19,20,30)$.

Generating tunnel entry. A $2 \mathrm{~cm}$, longitudinal mucosa incision is used to generate tunnel entry. A further submucosal dissection of $\geq 0.5 \mathrm{~cm}$ along the sides of the longitudinal incision is made to facilitate tumor extraction and gas diffusion $(15,31)$. For SMTs $>35 \mathrm{~mm}$, the mucosal incision may be increased to the size of the short dimension of the tumor (32).

Generating the tunnel. A submucosal tunnel extending $2 \mathrm{~cm}$ from the tumor is generated between the submucosal and MP layers using the ESD method. The selection of ESD knives depends on surgical experience; available knives include dual, hybrid, triangular-tip and hook knives. The dissection plane should be maintained proximate to the MP to decrease the risk of injury to the mucosal flap. The tunnel should be sufficiently wide and its width should increase according to the diameter 


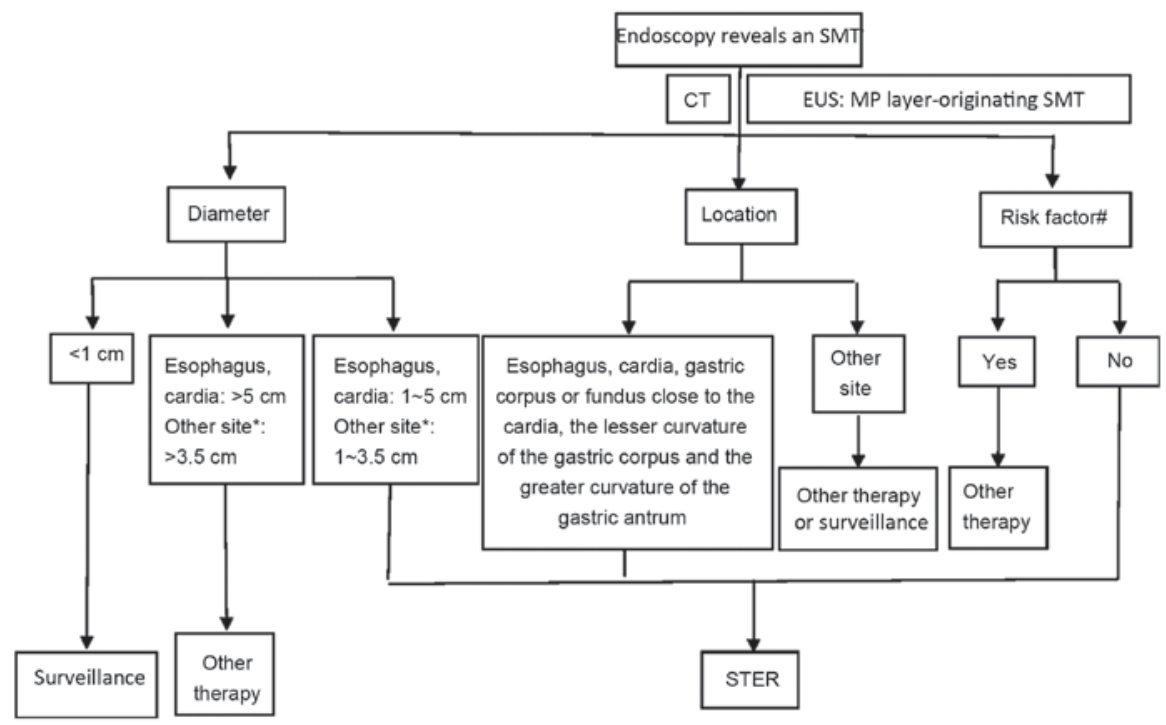

Figure 1. Patient selection diagram for candidates for STER at the Second Xiangya Hospital of Central South University (Changsha, China). *Gastric corpus or fundus proximate to the cardia, the lesser curvature of the gastric corpus and the greater curvature of the gastric antrum. "Ulceration or erosion at the tumor site; EUS reveals an irregular border, or internal heterogeneity, including an anechoic area (i.e. necrosis), echogenic loci (i.e. bleeding), heterogeneous enhancement or regional lymph node swelling; CT reveals metastasis or invasion out of the gastrointestinal tract; a Zubrod-Eastern Cooperative Oncology Group Performance Status $\geq 2$; patient exhibits severe cardiopulmonary disease or blood coagulation disorders. SMT, submucosal tumor; CT, computerized tomography; EUS, endoscopic ultrasonography; MP, muscularis propria; STER, submucosal tunneling endoscopic resection.

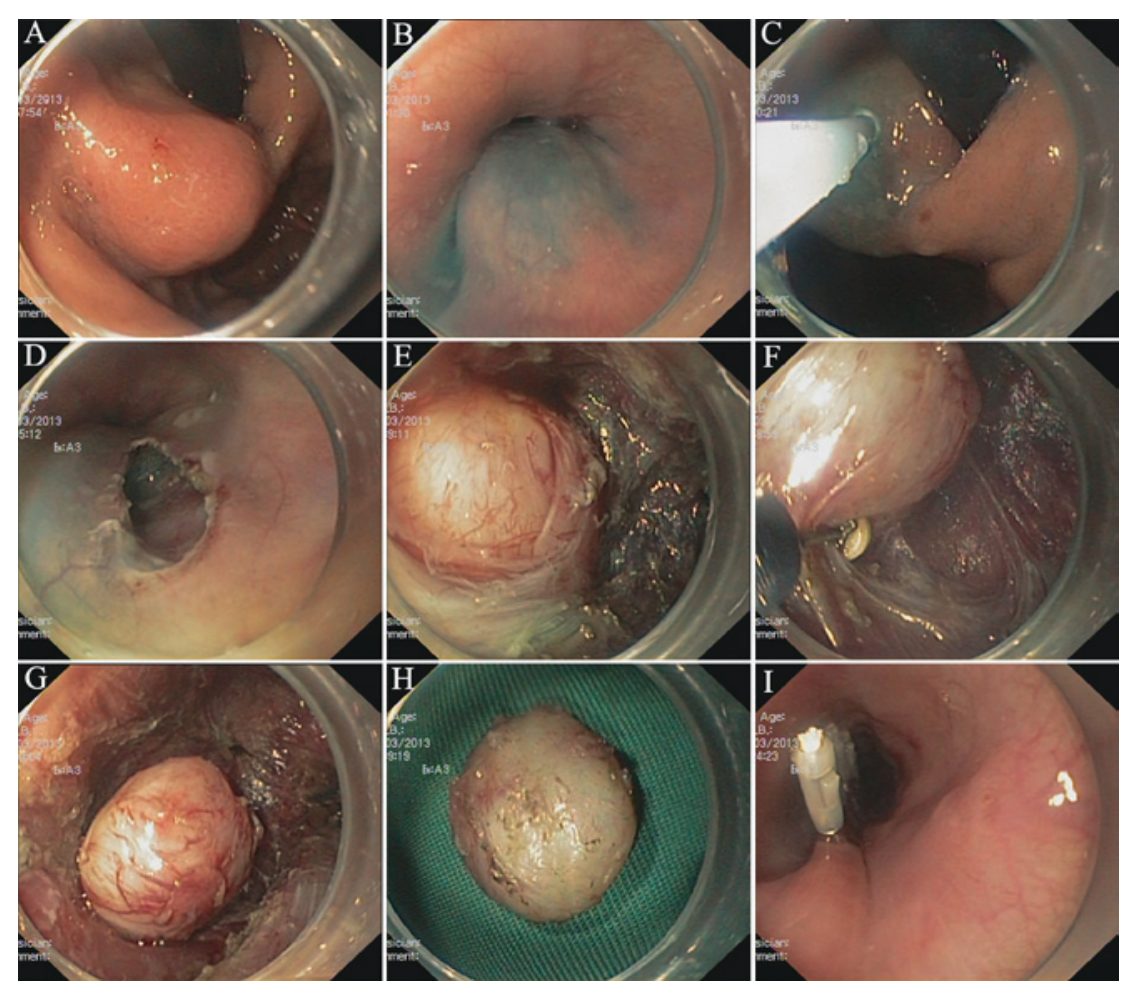

Figure 2. Submucosal tunneling endoscopic resection procedure in the Second Xiangya Hospital of Central South University (Changsha, China). (A) Submucosal tumor in the gastric fundus proximate to the cardia. (B) Submucosal injection. (C) The marked location of the tumor. (D) Tunnel entry. (E) The submucosal tumor. (F) Tumor dissection. (G) The dissected tumor in the tunnel. (H) The resected tumor. (I) Mucosal entry closure.

of the SMT to ensure a satisfactory endoscopic view of the SMT and sufficient space for resection and facilitate submucosal dissection and gas diffusion (32).

Dissection of the tumor. The tumor is dissected at the MP layer. Complete resection without damaging the tumor capsule is recommended. For SMTs originating from the deep MP layer or exhibiting a tight connection with the underlying MP or serosal layers, a full-thickness resection, including the lesion, its underlying MP and serosa is generally performed $(10,15,32)$. For patients with gastric SMTs undergoing full-thickness resection, the SMT should be prevented from lodging in the 
abdominal cavity, potentially by using laparoscopic assisted endoscopic surgery.

Removing the tumor. Although small SMTs may be easily removed from the tunnel and extracted from the body, doing so for SMTs $>35 \mathrm{~mm}$ in the upper gastrointestinal tract may prove challenging. While removing SMTs from the upper gastrointestinal tract, the tumor is grasped such that its long dimensions are respectively parallel and transverse to the long axis of the esophagus, and the tumor may be easily extracted through the tunnel orifice and the upper esophageal sphincter (32). If preoperative imagery, endoscopy and clinical examination suggest a benign tumor, a snare may be used following the completion of resection to cut the tumor while still in the tunnel into $\geq 2$ pieces to facilitate its extraction from the tunnel (32). An alternative approach is to generate a second 'window', either in the area of the tumor or through a distal mucosal incision to facilitate en bloc extraction for large leiomyomas $(24,26)$.

Closing the tunnel entry. Following the removal of the SMT, the wound surface should be repeatedly washed to decrease the risk of residual tumor cells. Subsequently, several clips are applied to close the tunnel entry.

Managing the resected tumor. The specimens are then fixed, embedded with paraffin, and sectioned. Hematoxylin and eosin and immunohistochemical staining are performed to detect cluster of differentiation (CD) 34, CD117, S100 calcium binding proteins, desmin, survival of motor neuron 1, marker of proliferation Ki-67 and gastrointestinal stromal tumor 1.

Postoperative management. The postoperative symptoms of patients are monitored, including fever, chest or abdominal pain, dyspnea, cyanosis, distention and peritonitis. Thoracoabdominal radiography, second look endoscopy or CT is performed for selected patients with postoperative symptoms 2 days following the operation. Generally, patients are kept nil per os for $24 \mathrm{~h}$, subsequently placed on a liquid diet for multiple days to a week, and gradually returned to a normal diet following this. Intravenous antibiotics and potentially hemostatics are administered to patients for 3 days. For patients with upper gastrointestinal SMTs, intravenous proton pump inhibitors are administered for 3-7 days and orally administered for multiple weeks following this. For rectal SMTs, it is necessary to ensure stools remain soft and defecation easy (30).

\section{Efficacy of STER}

Currently, $>20$ studies have been published with outcome data based on $>700$ patients $(6-20,22,29,30,32-35)$. In these studies, therapeutic success was recorded for $>77 \%$ of patients and en bloc resection was achieved in $>85 \%$ of patients, while irregularly shaped or larger tumors were risk factors in piecemeal resection (33). Of the SMTs reported in these studies, $>95 \%$ were leimyomas or gastrointestinal stromal tumors, while the other reported tumors included lipomas, schwannomas, calcifying fibrous, glomus, granular cell and nerve sheath tumors, proliferating collagen fibers, degenerated nodes and aberrant pancreatic tissue. No recurrence was detected for the patients of these studies. To the best of our knowledge, there are no randomized, controlled trials comparing STER with other treatments of SMTs, but three retrospective studies have been published.

Comparing STER and ESD. Wang et al (34) retrospectively assessed the clinical data of 39 patients with esophageal leiomyoma, 18 of which received STER and 21 of which received ESD, and demonstrated that the efficacy and complications of the two techniques were comparable, though STER was associated with decreased operating time and duration of hospital stay, and an increased rate of incision healing compared with ESD.

Comparing STER and ESE. Lu et al (35) retrospectively analyzed the clinical data of 77 patients with upper gastrointestinal SMTs, 42 of which received STER and 35 of which received ESE, and demonstrated that the efficacy and complications of the two techniques were comparable, though STER decreased air leakage for SMTs by $>10 \mathrm{~mm}$ compared with ESE.

Comparing STER and video-assisted thoracoscopic surgery (VATS). Tan et al (32) retrospectively evaluated the clinical data of 31 patients with esophageal leiomyoma (diameter, 35-55 mm), 18 of which received STER and 13 of which received VATS, and revealed that the efficacy of the two techniques were comparable, though STER was associated with decreased operation time, a reduced decrease in hemoglobin level, and decreased cost and duration of hospital stay compared with VATS.

\section{Complications of STER}

In the aforementioned $>20$ studies, STER has been performed with a decreased rate of serious complications, and no STER-associated mortality has been reported. Nonetheless, efforts should be taken to decrease the risk of adverse events, recognize them when they occur, and manage them appropriately following identification. According to a previously published, large-scale study consisting of 290 patients with SMTs who underwent STER, the overall incidence of complications was $23.4 \%$ (68/290), and only $10.0 \%$ of procedures (29/290) required intervention for complications (29). Furthermore, the study demonstrated that irregular shape, the location of the tumor in the deep MP layer, increased procedure time, and air insufflation were risk factors for major STER-associated complications.

\section{Intraoperative complications}

Aspiration. An important consideration in the use of STER is the risk of aspiration during induction and intubation, and communicating with the anesthesiologist is crucial to decrease this. Standard airway protection methods should be used, including a rapid induction sequence, to decrease the risk of aggressive aspiration of mouth contents during intubation.

Bleeding. Bleeding may occur at any time during STER, but usually results in $<100 \mathrm{ml}$ blood loss and may be immediately 
controlled via coagulation with the tip of a knife. However, the availability of electrosurgical hemostatic forceps for the coagulation of larger vessels is essential. Chen et al (29) reported a rate of $1.7 \%$ (5/290) for major bleeding (>200 ml). All of those cases were managed endoscopically and blood transfusion was not required.

Mucosal laceration. Mucosal lacerations may occasionally occur and the majority are small $(<1 \mathrm{~cm})$ and may be closed using $\geq 1$ clip. In the aforementioned $>20$ studies, mucosal laceration occurred in a total of 15 patients and in each case the laceration was closed using clips without leakage $(10,15,16,18,19,29)$. Two case reports have reported on large esophageal mucosal lacerations, which were managed using stent insertions $(26,28)$.

Gas-associated complications. Gas-associated complications include subcutaneous emphysema, pneumothorax, pneumoperitoneum, and mediastinal emphysema. Gas-associated complications are the most common complications of STER and may occur in $\leq 66.7 \%$ of patients undergoing STER (10). Those patients who undergo full-thickness resection exhibit an increased rate of gas-associated complications, though the majority of these are clinically insignificant and may resolve spontaneously $(7,10,12,13,29)$. Thoracic drainage is recommended for pneumothorax in patients with lung collapse $>30 \%$ and symptoms that include dyspnea, and lung puncture is recommended for patients with pneumoperitoneum or emphysema and more apparent symptoms (29). $\mathrm{CO}_{2}$ is recommended as the insufflation gas instead of air. For $\mathrm{CO}_{2}$ insufflator set-ups that allow adjustments to $\mathrm{CO}_{2}$ flow, the lower flow setting should be set once the submucosal tunnel, and particularly the MP, is breeched. Regardless of whether an adjustable $\mathrm{CO}_{2}$ insufflator is used, the endoscopist should use insufflation sparingly while in the submucosal tunnel. In all the aforementioned studies, STER was not discontinued for any patients exhibiting intraoperative complications.

\section{Postoperative complications}

Fistula. The most challenging potential complication of STER is leakage from the associated fistula. In $>700$ patients undergoing STER that have been described, leaks were uncommon, and no leak-associated mortalities have been reported. Only one leak (esophageal-pleural fistula; $<0.2 \%$ ) was reported and it was managed using clips and thoracic drainage (29).

Infection. Infection includes mediastinitis, peritonitis, subphrenic and intra-tunnel infections, and symptoms include chest/abdominal pain and a fever $>38^{\circ} \mathrm{C}$. In all the aforementioned $>20$ studies, significant infection was uncommon, and mediastinitis, subphrenic and intra-tunnel infections have been reported in 1, 1 and 2 patients, respectively (14,16,29). All the reported cases of infection were controlled through conservative management, and no infection-associated mortalities have been reported.

Pleural or mediastinal effusion. The majority of effusions are reactive, but may be considered a normal postoperative change and, as with peroral endoscopic myotomy for treating patients with achalasia (36), clinically significant effusions are uncommon. In the $>700$ patients undergoing STER that have been described, $16(2 \%)$ and 1 exhibited clinically significant pleural and mediastinal effusion, respectively, and these cases of effusion were treated using antibiotics and/or drainage $(10,13,14,29)$.

Bleeding. Although postoperative bleeding is a potential concern, no cases of postoperative bleeding have been reported in the aforementioned $>20$ studies. Other, rare complications that have been anecdotally presented and may be study-dependent and of decreased general significance include wound pain and diverticulum formation $(15,16,29)$.

\section{Conclusions}

It is estimated that $>1,000$ STERs have been performed globally over the last 5 years (37). The previous studies that reported the outcomes of $>700$ STERs (mean follow up, 3.5-22.7 months), demonstrating an en bloc resection rate of $85.7-100 \%$, negligible severe morbidity, and no mortality or recurrence. In addition, $\leq 10 \%$ of the patients enrolled in these studies exhibited intervention-requiring complications. These favorable outcomes suggested that STER may represent a promising treatment for patients with SMTs. However, STER remains a complicated endoscopic surgery that requires a multidisciplinary team with expertise in surgery and advanced endoscopy, and the patients for which STER is performed should be selected carefully.

\section{References}

1. Nishida T, Kawai N, Yamaguchi S and Nishida Y: Submucosal tumors: Comprehensive guide for the diagnosis and therapy of gastrointestinal submucosal tumors. Dig Endosc 25: 479-489, 2013.

2. Kim GH: Endoscopic resection of subepithelial tumors. Clin Endosc 45: 240-244, 2012.

3. Zhou PH, Yao LQ, Qin XY, Cai MY, Xu MD, Zhong YS Chen WF, Zhang YQ, Qin WZ, Hu JW and Liu JZ: Endoscopic full-thickness resection without laparoscopic assistance for gastric submucosal tumors originated from the muscularis propria. Surg Endosc 25: 2926-2931, 2011.

4. Shi Q, Zhong YS, Yao LQ, Zhou PH, Xu MD and Wang P: Endoscopic submucosal dissection for treatment of esophageal submucosal tumors originating from the muscularis propria layer. Gastrointest Endosc 74: 1194-1200, 2011

5. Zhang Y, Ye LP, Zhu LH, Zhou XB, Mao XL and Ding JX: Endoscopic muscularis excavation for subepithelial tumors of the esophagogastric junction originating from the muscularis propria layer. Dig Dis Sci 58: 1335-1340, 2013.

6. Inoue H, Ikeda H, Hosoya T, Onimaru M, Yoshida A, Eleftheriadis N, Maselli R and Kudo S: Submucosal endoscopic tumor resection for subepithelial tumors in the esophagus and cardia. Endoscopy 44: 225-230, 2012.

7. Xu MD, Cai MY, Zhou PH, Qin XY,Zhong YS, Chen WF, Hu JW, Zhang YQ, Ma LL, Qin WZ and Yao LQ: Submucosal tunneling endoscopic resection: A new technique for treating upper GI submucosal tumors originating from the muscularis propria layer (with videos). Gastrointest Endosc 75: 195-199, 2012.

8. Gong W, Xiong Y, Zhi F, Liu S, Wang A and Jiang B: Preliminary experience of endoscopic submucosal tunnel dissection for upper gastrointestinal submucosal tumors. Endoscopy 44: 231-235, 2012.

9. Ye LP, Zhang Y, Mao XL, Zhu LH, Zhou XB, He SQ, Chen JY and Jin X: Submucosal tunnelling endoscopic resection for the treatment of esophageal submucosal tumours originating from the muscularis propria layer: An analysis of 15 cases. Dig Liver Dis 45: 119-123, 2013. 
10. Liu BR, Song JT, Kong LJ, Pei FH, Wang XH and Du YJ: Tunneling endoscopic muscularis dissection for subepithelial tumors originating from the muscularis propria of the esophagus and gastric cardia. Surg Endosc 27: 4354-4359, 2013.

11. Jiao CH, Yang SP, Li XL, Ding J, Xu YH, Tao G, Chen L, Zhang DQ, He X, Chen WK and Shi RH: Preliminary exploration on submucosal tunneling endoscopic resection for middle and lower esophagus submucosal tumors. Zhonghua Yi Xue Za Zhi 93: 2388-2391, 2013 (In Chinese).

12. Ye LP, Zhang Y, Mao XL, Zhu LH, Zhou X and Chen JY: Submucosal tunneling endoscopic resection for small upper gastrointestinal subepithelial tumors originating from the muscularis propria layer. Surg Endosc 28: 524-530, 2014

13. Wang XY, Xu MD, Yao LQ, Zhou PH, Pleskow D, Li QL, Zhang YQ, Chen WF and Zhong YS: Submucosal tunneling endoscopic resection for submucosal tumors of the esophagogastric junction originating from the muscularis propria layer: A feasibility study (with videos). Surg Endosc 28: 1971-1977, 2014.

14. Zhou DJ, Dai ZB, Wells MM, Yu DL, Zhang J and Zhang L: Submucosal tunneling and endoscopic resection of submucosal tumors at the esophagogastric junction. World J Gastroenterol 21: 578-583, 2015.

15. Wang H, Tan Y, Zhou Y, Wang Y, Li C, Zhou J, Zhang J and Liu D: Submucosal tunneling endoscopic resection for upper gastrointestinal submucosal tumors originating from the muscularis propria layer. Eur J Gastroenterol Hepatol 27: 776-780, 2015.

16. Zhao H, Sheng H, Huang L, Jiang L, Xie Y and Zhou P: Submucosal tunneling endoscopic resection in the treatment of esophageal submucosal tumors originating from muscularis propria layer. Zhonghua Wei Chang Wai Ke Za Zhi 18: 478-482, 2015 (In Chinese).

17. Li B, Liu J, Lu Y, Hao J, Liu H, Jiang J, Jiang Y, Qin C and $\mathrm{Xu} \mathrm{H}$ : Submucosal tunneling endoscopic resection for tumors of the esophagogastric junction. Minim Invasive Ther Allied Technol 25: 141-147, 2016.

18. Lu J, Zheng M, Jiao T, Wang Y and Lu X: Transcardiac tunneling technique for endoscopic submucosal dissection of gastric fundus tumors arising from the muscularis propria. Endoscopy 46 : 888-892, 2014

19. Lu J, Jiao T, Li Y, Liu Y, Wang Y, Wang Y, Zheng M and Lu X: Heading toward the right direction-solution package for endoscopic submucosal tunneling resection in the stomach. PLoS One 10: e0119870, 2015.

20. Li QL, Chen WF, Zhang C, Hu JW, Zhou PH, Zhang YQ, Zhong YS, Yao LQ and Xu MD: Clinical impact of submucosal tunneling endoscopic resection for the treatment of gastric submucosal tumors originating from the muscularis propria layer (with video). Surg Endosc 29: 3640-3646, 2015.

21. Chen H, Xu Z, Huo J and Liu D: Submucosal tunneling endoscopic resection for simultaneous esophageal and cardia submucosal tumors originating from the muscularis propria layer (with video). Dig Endosc 27: 155-158, 2015.

22. Zhang C, Hu JW, Chen T, Zhou PH, Zhong YS, Zhang YQ, Chen WF, Li QL, Yao LQ and Xu MD: Submucosal tunneling endoscopic resection for upper gastrointestinal multiple submucosal tumors originating from the muscular propria layer: A feasibility study. Indian J Cancer 51 (Suppl 2): e52-e55, 2015.

23. Tan Y and Liu D: En bloc submucosal tunneling endoscopic resection for a giant esophageal leiomyoma. Gastrointest Endosc 82: 399, 2015 .
24. Ng JJ, Chiu PW, Shabbir A and So JB: Removal of a large, 40-mm, submucosal leiomyoma using submucosal tunneling endoscopic resection and extraction of specimen using a distal mucosal incision. Endoscopy 47 (Suppl 1): E232-E233, 2015.

25. Maydeo A, Sharma A, Bhandari S and Dhir V: Submucosal tunneling and endoscopic resection of a large, esophageal leiomyoma. Gastrointest Endosc 82: 954, 2015.

26. Tan Y, Zhu H, Lv L and Liu D: Enlarging an accidental mucosotomy to facilitate tumor extraction during submucosal tunneling endoscopic resection for a giant esophageal leiomyoma. Gastrointest Endosc 83: 248-249, 2016.

27. Liu H, Wei LL, Zhang YZ, Sha QM, Huang Y, Qin CY and Xu HW: Submucosal tunnelling endoscopic resection (STER) for the treatment of a case of huge esophageal tumor arising in the muscularis propria: A case report and review of literature. Int J Clin Exp Med 8: 15846-15851, 2015.

28. Kumbhari V, Saxena P, Azola A, Messallam AA, El Zein MH and Khashab MA: Submucosal tunneling endoscopic resection of a giant esophageal leiomyoma. Gastrointest Endosc 81: 219-220, 2015.

29. Chen T, Zhang C, Yao LQ, Zhou PH, Zhong YS, Zhang YQ Chen WF, Li QL, Cai MY, Chu Y and Xu MD: Management of the complications of submucosal tunneling endoscopic resection for upper gastrointestinal submucosal tumors. Endoscopy 48: 149-155, 2016.

30. Hu JW, Zhang C, Chen T, Zhou PH, Zhong YS, Zhang YQ, Chen WF, Li QL, Yao LQ and Xu MD: Submucosal tunneling endoscopic resection for the treatment of rectal submucosal tumors originating from the muscular propria layer. J Cancer Res Ther 10 (Suppl): S281-S286, 2014.

31. Wang X, Tan Y, Zhang J and Liu D: Risk factors for gas-related complications of peroral endoscopic myotomy in achalasia. Neth J Med 73: 76-81, 2015.

32. Tan Y, Lv L, Duan T, Zhou J, Peng D, Tang Y and Liu D Comparison between submucosal tunneling endoscopic resection and video-assisted thoracoscopic surgery for large esophageal leiomyoma originating from the muscularis propria layer. Surg Endosc 30: 3121-3127, 2016.

33. Chen T, Zhou PH, Chu Y, Zhang YQ, Chen WF, Ji Y, Yao LQ and $\mathrm{Xu}$ MD: Long-term outcomes of submucosal tunneling endoscopic resection for upper gastrointestinal submucosal tumors. Ann Surg 265: 363-369, 2017.

34. Wang L, Ren W, Zhang Z, Yu J, Li Y and Song Y: Retrospective study of endoscopic submucosal tunnel dissection (ESTD) for surgical resection of esophageal leiomyoma. Surg Endosc 27: 4259-4266, 2013.

35. Lu J, Jiao T, Zheng M and Lu X: Endoscopic resection of submucosal tumors in muscularis propria: The choice between direct excavation and tunneling resection. Surg Endosc 28: 3401-3407, 2014.

36. Yang S, Zeng MS, Zhang ZY, Zhang HL, Liang L and Zhang XW: Pneumomediastinum and pneumoperitoneum on computed tomography after peroral endoscopic myotomy (POEM): Postoperative changes or complications? Acta Radiol 56: 1216-1221, 2015.

37. Lv X, Wang CH and Xie Y: Efficacy and safety of submucosal tunneling endoscopic resection for upper gastrointestinal submucosal tumors: A systematic review and meta-analysis. Surg Endosc 31: 49-63, 2017. 